\title{
Tensiones identitarias y lógicas adaptativas en la oferta de senvicios agrícolas pampeanos (2009-2015)
}

\author{
Tensions of Identity and Adaptive Logics in the Supply of Agricultural Services in the Pampa (2009-2015).
}

\author{
José Muzlera \\ CONICET. Centro de Estudios de la Argentina Rural. \\ Universidad Nacional de Quilmes, Argentina \\ jmuzlera2000@gmail.com \\ Rocío Pérez Gañán \\ CONICET. Centro de Estudios de la Argentina Rural. \\ Universidad Nacional de Quilmes, Argentina \\ mr.perez@ucm.es
}

\begin{abstract}
Resumen:
Los prestadores de servicios de maquinaria agrícola -contratistas- realizan las labores culturales para la agricultura extensiva de la región pampeana argentina. A través de una encuesta de alcance nacional a 426 contratistas complementada con entrevistas cualitativas, se ha caracterizado el tipo de estructura, los servicios ofrecidos, el nivel de capitalización y la dimensión familiar de sus empresas. Los resultados obtenidos muestran la pérdida del peso explicativo de la identidad de estos agentes del agro frente al creciente volumen y flexibilidad del capital, y el peso de la dimensión familiar para definir las dinámicas empresariales en la Región Pampeana Argentina.
\end{abstract}

Palabras Clave: Contratistas, Agronegocio, Pluriactividad, Empresas familiares.

\begin{abstract}
:
Those offering machinery services in the agricultural sector (contractors) are entrepreneurs who carry out the majority of cultural labor for the extensive agriculture of the Argentine region of the Pampa. In this context, and by way of a national poll of 426 such contractors, complemented with qualitative interviews, we have tried to characterize the type of structure, the services offered, the level of capitalization, and the family dimension of their companies. The obtained results highlight two great issues. First, the loss of explanatory weight that identity has in the behavior of these agents in opposition to the volume and flexibility of capital. Second, the importance of the family dimension to defining business dynamics in the Pampa.
\end{abstract}

KEYWORDS: Contractors, Agribusiness, Pluriactivity, Family businesses.

\section{INTRODUCCIÓN}

En la Argentina, "lo agrario" (fuertemente vinculado a "lo rural") se ha conformado inequívocamente como un sector de importancia fundamental en la construcción y articulación de la vida social, económica y política del país. En este espacio rural se ha desarrollado un proceso de modernización capitalista -iniciado en la segunda mitad de los años setenta e intensificado durante los años noventa en el marco de unas políticas neoliberales-, que acabó consolidando, al comienzo del siglo XXI, un modelo de desarrollo capitalista del sector agrario conocido como agronegocio (Paz, 2017; Kay, 2016; Gras y Hernández, 2013, 2016). En este modelo de desarrollo el contratista se erige como un actor social agrario relevante sobre el que surgen preguntas específicas sobre la vertiginosidad de sus transformaciones y su capacidad de adaptación (Lombardo y Marra, 2018; Fernández, 2017, 2013; Muzlera, 2016, 2013; Villulla, 2015; Stadler y Botta, 2015; Azcuy Amehino, 2009). 
Desde el campo de la sociología rural, los estudios realizados se han centrado en reflejar la situación de los sujetos-actores en relación con las problemáticas más generales que los afectan, como son los procesos de expansión productiva, transformaciones en las formas y en la organización del trabajo, ${ }^{1}$ etc. No obstante, el enfoque de este artículo se centra en la necesidad de considerar no solo las trasformaciones acaecidas en un nivel objetivo, sino aquellas que se producen en el plano subjetivo donde los cambios de oferta y demanda en el mercado y el avance tecnológico han influido en la cotidianidad de las dinámicas familiares, al mismo tiempo que dichas dinámicas familiares han llegado a caracterizar el tipo de oferta. Se ha materializado así una variedad de formas de acuerdo y toma de decisiones en las trayectorias seguidas (Muzlera, 2016; Craviotti y Gras, 2006). Siguiendo esta línea, este artículo ha tratado de visibilizar la complejidad de categorización de los contratistas contemporáneos -debido a su heterogeneidad y diversidad en constante aumento-, y ha intentado profundizar en sus racionalidades para comprender el modo en que se comportan como clase cuando la actividad, propiamente dicha, ya no define a este sujeto-actor del agro (Varela y Cappello, 2018; Kahneman, 2003; Bourdieu, 1998).

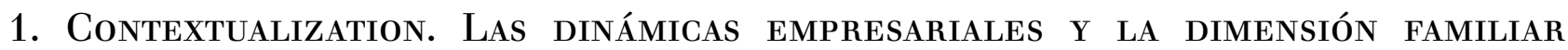 PAMPEANA: UNA APROXIMACIÓN DESDE LA COMPLEJIDAD DE LA FLEXIBILIZACIÓN EN EL MERCADO}

El agronegocio, nuevo paradigma hegemónico agrario, tiende -entre otras particularidades-, a homogeneizar procesos productivos, mientras heterogeneiza a los sujetos y sus estrategias, lo que genera una relación de continuidad entre el cambio material (tecnologías) y el subjetivo (mentalidades) (Gras y Hernández, 2016, p. 263). Como parte de este proceso, en el cual los sujetos del paradigma anterior deben adaptarse o desaparecer, los contratistas crecen en número y en importancia. ${ }^{2}$ A pesar de que puede encontrarse evidencia de que estas empresas existen en el país hace un siglo y medio, es necesario llegar a la década de los ochenta del siglo XX para encontrar los primeros trabajos e investigaciones sobre su definición y caracterización (Llovet, 1991; Devoto, 1988, 1989; Tort, 1983; Baumeister, 1980; Pizarro y Cacciamani, 1980). Posteriormente, si se siguen los trabajos de Forni y Tort (1991), puede observarse que los contratistas de servicios se erigen como mediadores eficientes entre los avances tecnológicos que van surgiendo y su implementación en la producción agraria. En este sentido, en relación a la Pampa Húmeda, el contratismo va a conformarse como un modelo de trabajo unido al desarrollo de la agricultura cerealera extensiva pampeana desde sus orígenes (Barsky y Pucciarelli, 1997), cuyo auge y relevancia está relacionado con la intensificación y el proceso de mecanización y la "democratización" en el acceso a niveles tecnológicos más avanzados de esta agricultura extensiva (Tort, 1983). Complementariamente, son considerados como los facilitadores en la provisión de maquinaria para el mantenimiento de la producción de muchos productores sin recursos suficientes (Villulla, 2015; Muzlera, 2009; Craviotti, 2002; Pizarro y Cacciamani, 1980).

Forni y Tort (1991), en su categorización de contratistas, distinguían dos grupos principales: aquellos denominados contratistas de servicios, que ofrecen servicios a terceros de laboreo, siembra, protección de cultivos y cosecha, y reciben como pago por su prestación dinero y/o especies; y los llamados contratistas de producción o tanteros, cuyo pago es realizado a través de porcentajes de la producción de terceros mediante contratos (orales/escritos) y asumen los posibles riesgos de la actividad (Stadler y Botta, 2015; Forni y Tort, 1991). Esta categorización fue complejizada por Lódola, Angeletti y Fossati (2005) a partir de un trabajo netamente cuantitativo en el que distinguían entre contratistas puros, contratistas-productores y productores-contratistas, pero no tenían en cuenta el rol de las identidades de estos empresarios, sino la actividad que produjese mayores ingresos. Luego de retomar estos y otros trabajos anteriores, Muzlera (2013) complejiza la categoría considerando las identidades y habitus de estos empresarios. Finalmente, pocos años después, Hernández y Muzlera (2016), Varela y Cappello (2018) y Lombardo y Marra (2018) repiensan estas categorizaciones considerando no solo la diversificación o no de sus actividades, sino también su movilidad 
geográfica, su formación y capacidades, sus finanzas y sus formas de comerciar. De este modo, los autores visibilizan una complejidad cada vez más presente en el agro pampeano en la que los contratistas de servicios "no constituyen un conjunto socio-profesional con características homogéneas ni a nivel de su origen social ni de las trayectorias de su formación ni, incluso, en relación al tipo de organización de su trabajo (individual, empresarial)" (Hernández y Muzlera, 2016, p. 2), sino sujetos flexibles y flexibilizados por los imperativos del mercado y la lógica del agronegocio, en la que van acumulando posibilidades potenciales a medida que incrementan el riesgo, pierden capacidad de agencia y ven corroído su carácter (Sennett, 2000). De esta forma, aparecen tanto contratistas que diversifican su cartera de servicios como quienes se especializan en una sola actividad (semillas, siembra, cosecha o fumigación), quienes son nómades o quienes no se mueven de su lugar de residencia; quienes ven en la actividad un valor y para quienes solo es un negocio; quienes se consideran contratistas, pero también toman a su cargo producción, y quienes son productores que también venden servicios.

La tercerización de labores es uno de los soportes del agronegocio en Argentina (Paz, 2017; Kay, 2016; Gras y Hernández, 2009). Esta modalidad es tan extendida que otros lugares del mundo la llaman "modo de producción a la Argentina”. Desde los primeros trabajos encontrados sobre contratistas, mencionados anteriormente, hasta los más recientes (Piñeiro, Robles y Elverdin, 2017; Ullua, 2017; Fernández, 2017; Garbers, 2016; Hernández y Muzlera, 2016; Villulla, 2015; Urcola, De Sartre, Veiga, Elverdin y Albadalejo, 2015; Lódola, 2008; Agüero, Rivarola y Maldonado, 2007; entre otros), el sector se ha transformado radicalmente. ${ }^{3}$ En el presente trabajo se han retomado temáticas abordadas en trabajos previos (Hernández y Muzlera, 2016; Villulla, 2015; Lódola, Angeletti y Fossati, 2005), como las dinámicas empresariales y la dimensión familiar, para profundizarlas y abordarlas desde una perspectiva particular: la oferta de labores culturales ${ }^{4}$ para la agricultura extensiva.

\section{ConSIDERACIONES METODOLÓGicas}

El enfoque de este trabajo es eminentemente cuantitativo y ha sido desarrollado a partir de una encuesta nacional ${ }^{5}$ realizada a 426 empresarios y exempresarios prestadores de servicios de maquinaria agrícola de la República Argentina entre marzo y diciembre de 2015. A pesar del carácter cuantitativo del análisis, esta investigación no ha renunciado a visibilizar las voces de los protagonistas en un ejercicio de complementariedad discursiva con los datos analizados (Van Dijk, 2003). Para ello, se llevó a cabo un extenso trabajo de campo (Hammersley, Martyn y Atkinson, 1994; Guber, 2001) en el que se realizaron entrevistas semiestructuradas (Valles, 2002) a miembros de 46 empresas contratistas de maquinaria agrícola de la Región Pampeana entre 2009 y 2014.

En relación con la metodología cuantitativa, la muestra final (426 casos) estuvo compuesta por un 63,2 $\%$ de contratistas que pertenecen a alguna agrupación o institución que los nuclea. La encuesta contenía 75 preguntas que relevaban distintas dimensiones temáticas (familia, calidad de vida, dificultades empresariales, otras actividades, niveles de capitalización, formación societaria, cantidad y perfil de demandantes, vínculos y tensiones empresa-familia y desplazamiento espacial entre otros). La recolección de datos fue financiada por Proyecto de Investigación Plurianual (PIP) CONICET “Economías Regionales, Burocracia, Tecnología y Medio Ambiente".

Respecto al trabajo cualitativo, las sucesivas estadías en campo (continuas entre 2009 y 2014) fueron producto de proyectos de investigación puntuales. Las entrevistas trataron de reconstruir trayectorias profesionales e historias de vida que permitiesen comprender las subjetividades y racionalidades puestas en juego al momento de organizar el trabajo y desarrollar estrategias de capitalización. El análisis de las entrevistas se complementó con observaciones llevadas a cabo en asambleas, explotaciones en actividad, talleres de reparación de maquinarias y casas de familia, asados y otros eventos. La selección de los prestadores 
de servicios se realizó principalmente mediante la técnica de "bola de nieve", es decir, en función de un listado conformado a partir de contactos ofrecidos por otros informantes. Esta "bola de nieve" se inició en cuatro lugares distintos: a) el sudeste de la provincia de Buenos Aires (partidos de Balcarce y Mechongué); b) el sur y centro de la provincia de Santa Fe (departamento de Castellanos, San Jerónimo y San Justo); c) el sur de la provincia de Buenos Aires (partido de Saavedra); y d) el centro bonaerense (Saladillo y 25 de Mayo). De este modo se dio un poco más de representatividad y heterogeneidad a la muestra. Se ha recurrido a la complementariedad de técnicas cualitativas por entender que las narrativas de los propios protagonistas juegan un papel fundamental en la configuración de los discursos, representaciones e interacciones que (re)crean la cuestión del agro y que son útiles para la interpretación de los datos que arrojó la encuesta.

\section{Resultados. Tribulaciones y lógicas adAPtativas de lOS CONTRATistas Pampeanos EN SIGLO XXI}

Las empresas prestadoras de servicios suelen ser empresas familiares capitalizadas. ${ }^{6}$ Trabajos anteriores (Lódola, Angeletti y Fossati, 2005; Muzlera, 2013) diferencian entre "contratistas puros", aquellos que solo se dedican a la venta de servicios, y "contratistas-productores", aquellos que realizan las dos actividades. De las 426 empresas familiares encuestadas, ${ }^{7}$ el $63 \%$ fueron contratistas productores y el $37 \%$ contratistas puros. En el agro de la modernidad tardía (Muzlera, 2013), donde la lógica del agronegocio se ha convertido en hegemónica, las identidades de los sujetos tienden a ser cada vez más flexibles y yuxtapuestas (Varela y Cappello, 2018; Lombardo y Marra, 2018; Piñeiro, Robles y Elverdin, 2017). Este "nuevo" agro, resultado de distintos procesos sociales a escala global y con particularidades locales, no tiene una fecha de origen definida, pero suele haber cierto acuerdo respecto a que las reformas neoliberales de comienzos de la década de $1990 \mathrm{y}$ la liberación de la soja transgénica en el mercado argentino en 1996 son una serie de puntos de partida (Gras y Hernández, 2016; Muzlera, 2013).

Históricamente, por ser las maquinarias que utilizan las de costos más elevados y por ser las más difíciles de amortizar para los productores (eran las que en menos jornadas anuales se requerían), es más factible que quienes ofrecían servicios de cosecha fueran contratistas puros. Esta particularidad, muy clara hace 30 o 40 años, va desdibujándose. Durante las décadas de 1960, 1970 y 1980 era común que mecánicos, empleados de industria metal mecánica y pequeños chacareros que decidían reconvertirse fuesen los que comprasen cosechadoras y se transformasen en contratistas (Muzlera, 2009). La mercantilización, que se intensifica en los 90, y las posibilidades que otorgó la devaluación de la moneda a comienzos del s. XXI dieron lugar a que muchas empresas dedicadas a la producción agrícola se diversificasen vendiendo servicios de siembra y pulverización (Muzlera, 2013). Después de la crisis sectorial que comenzó en 2008, y que según las zonas y las actividades duró hasta 2012 o 2015, con productores descapitalizados y empresas financieramente maltrechas, fue común que muchos contratistas de cosecha comenzaran a tomar a su cargo la producción agrícola, y le pagaran al dueño de la tierra un porcentaje (Muzlera, 2016). De esta manera, la diversificación (producción-venta de servicios) termina de instaurarse como "lo común". Por un lado, diversifican la cantidad de servicios que ofrecen (siembra, cosecha, pulverización, etc.), y, por otro, cada vez más empresas combinan la oferta de labores culturales con la de actividades de producción de granos o ganado. En 2015, estimaciones de la Dirección de Contratistas e Insumos de la Nación señalaban que estas empresas eran las responsables del $90 \%$ de la cosecha de granos, el $75 \%$ de las pulverizaciones y el $65 \%$ de la siembra. En esta línea, los datos obtenidos en la encuesta aplicada sugieren, también, que la oferta de cosecha es mayor que la del resto de los servicios: el 71,9\% de las empresas encuestadas vende servicios de cosecha, el 60,1\% de siembra, el $37,9 \%$ de pulverización y el $32,5 \%$ otro servicio.

Los modos de organización de estas empresas en formato o dinámica de pool -en la que el proceso productivo se fragmenta y distintas empresas/individuos toman a su cargo una o más partes del mismo en distintas unidades productivas-, típicos de esta etapa, favorecen que un individuo participe de varias 
empresas (con distintos roles en cada una de ellas, incluso con casos en los que un mismo sujeto es propietario en una y empleado en otra) y que cada empresa tome a su cargo distintas funciones en distintas opciones del negocio. Así, por ejemplo, un empresario A junto a un socio B gestionan e invierten en la siembra un lote de 250 ha de soja, pero las labores culturales las contratan. A su vez, A es socio de $\mathrm{C}$ con quien vende servicios de siembra (entre otros clientes a la unidad que conformó con B para hacer 250 ha de soja), la pulverización de esa soja la realiza $\mathrm{C}$ con otro socio $\mathrm{D}$ (a los cuales se les paga el servicio) y la cosecha la realiza otro contratista.

\subsection{La pluriactividad y la diversificación como elementos desconfiguradores de la identidad de los sujetos del agro pampeano}

La pluriactividad en tanto estrategia de supervivencia en explotaciones agropecuarias no es un proceso desconocido (López Castro y otros, 2011; Murmis, 1998), pero la novedad reside en que resulta cada vez más difícil establecer cuál es la actividad que los identifica. Como señalaba un entrevistado al ser interrogado sobre qué era y cuál era su actividad: "Yo soy todo y no soy nada... soy un empresario agropecuario" (Tano, comunicación personal, septiembre de 2016). Como muestra este relato, la superposición de actividades es tan amplia y tan dinámica que los sujetos no logran construir una identidad clara. Más de la mitad de los sujetos encuestados en este trabajo (el 55,4\%) antes de ser contratistas fueron productores agropecuarios, esta característica se intensifica entre quienes comenzaron a vender servicios en la década de 1990 (el 65,1 $\%$ fueron productores antes de vender servicios). Sin embargo, ser contratista no es algo que necesariamente sucede en el tiempo a ser productor, sino que, en general, es una superposición (como muestra el 62,1\% de los casos encuestados) (ver tabla 1). Otro proceso que se observa a través de los datos de esta investigación es que la diversificación de actividades sigue en aumento.

TABLA 1

Año de inicio de venta de servicios en función de si es (o no) productor

\begin{tabular}{|c|r|r|r|}
\hline Año en que comenzó a vender & \multicolumn{2}{|c|}{ ¿Es Productor agropecuario? \% } & \multirow{2}{*}{ Total } \\
\cline { 2 - 3 } servicios & Sí & No & $100,0(139)$ \\
\hline $1950-1991$ & 52,6 & 47,4 & 100,0 (161) \\
$1992-2001$ & 73,0 & 27,0 & $100,0(126)$ \\
$2002-2016$ & 59,3 & 40,7 & $100,0(426)$ \\
\hline
\end{tabular}

Fuente: Elaboración propia. ${ }^{*}$ Chi cuadrado de Pearson 0,063

El cuadro anterior corrobora lo ya mencionado respecto a la gran cantidad de contratistas-productores. Si bien esta diversificación es una práctica ampliamente difundida, podemos concentrarla en tres grupos: quienes comenzaron a vender servicios entre 1950 y 1991; quienes lo hicieron en la década de 1990, y quienes lo hicieron con posterioridad al 2001. De este modo, quienes empezaron a vender servicios entre $1950 \mathrm{y}$ 1991 y en la década de 1990 son, en general, los empresarios de mayor edad, algunos sin hijos que los sucedan en la empresa, y la mayoría con una relativa solvencia y estabilidad debido a su trayectoria. El 47,4 \% de los que comenzaron a vender servicios antes de 1991 son contratistas puros (y 52,6 \% contratistas productores). La diversificación, funcional a los agronegocios, se intensifica con estos. Acorde con estos nuevos tiempos, quienes comenzaron a vender servicios entre 1992 y 2001, el $73 \%$ son contratistas-productores y solo 27 $\%$ contratistas puros. Por último, entre los que comenzaron a vender servicios con posterioridad a 2002, el $37,9 \%$ solo venden servicios y el $62,1 \%$ son contratistas productores. Esto no se produce por una regresión en la tendencia, sino que se explica porque, con frecuencia, son empresarios más jóvenes -recién salidos de la empresa paterna-, que aún no han tenido oportunidad de capitalizarse e incursionar en la producción, 
pero casi todos planean hacerlo: "Todos tratamos de hacer un poco de todo. El agro de hoy te obliga a ir buscando las oportunidades. Además, es una forma de diversificar el riesgo" (Néstor, comunicación personal, noviembre 2015). La diversificación de actividades, como estrategia, se da no solo combinando la producción agropecuaria y la actividad contratista, sino que cada vez más hay empresas contratistas que ofrecen más de un servicio (ver tabla 2). El 66,3 \% de las empresas entrevistadas ofrece dos o más servicios.

TABLA 2

Cantidad de servicios que presta en función del año de inicio de la actividad

\begin{tabular}{|c|c|c|c|c|}
\hline & \multicolumn{2}{|c|}{$\begin{array}{l}\text { Cantidad de servicios que } \\
\text { presta } \%\end{array}$} & \multirow{2}{*}{ Total } \\
\hline & & 1 & 2 o más & \\
\hline \multirow{3}{*}{$\begin{array}{l}\text { Año en que comenzó a } \\
\text { vender servicios }\end{array}$} & $1950-1991$ & 43,3 & 56,7 & $100,0(136)$ \\
\hline & $1992-2001$ & 24,3 & 75,7 & $100,0(160)$ \\
\hline & $2002-2016$ & 35,2 & 64,8 & $100,0(128)$ \\
\hline Total & & 33,7 & 66,3 & $100,0(424)$ \\
\hline
\end{tabular}

Fuente: Elaboración propia. ${ }^{*}$ Chi cuadrado de Pearson 0,070

La lógica de diversificar servicios suele fundamentarse en tener trabajo todo el año para poder conservar mano de obra calificada que, de otro modo, quedaría sin ocupación, lo que haría probable que no estuviera disponible para la siguiente campaña. Como señala uno de los entrevistados:

Nosotros, en época de mi viejo, empezamos vendiendo servicios de siembra y arada (en aquel entonces había pocos contratistas por la zona). Después, juntamos unos pesitos y nos compramos una cosechadora que dejaba mucha más guita. Dejamos la siembra y el taller y llegamos a tener tres cosechadoras grandes. Pero después de la devaluación [se refiere a la salida de la convertibilidad en enero de 2002], las cosas cambiaron: se llenó de contratistas y de gente que no quiere laburar. ¡Conseguir un empleado que te vaya al campo cuesta un huevo! Hoy por hoy es casi imposible conseguir un tipo que te sepa laburar, que sea honesto y que vaya al campo... entonces, cuando conseguís uno, tenés que conservarlo. Si vos tenés solo cosechadoras necesitás más empleados y cuando termina la temporada no podés pagarles para que no hagan nada, entonces los tenés que mandar a su casa y capaz que unos meses después ya no te vuelven. En cambio, si vos tenés varias máquinas ${ }^{8}$, necesitás menos empleados y podés darles laburo todo el año. (Francisco, comunicación personal, noviembre 2015)

$\mathrm{Al}$ igual que sucede en la pluriactividad de servicios-producción, en lo que respecta a la diversificación de servicios también los más jóvenes se diversifican menos porque aún no tienen suficiente capitalización. No obstante, los datos muestran que, en los tres periodos agrupados de oferta de servicios, la tendencia de vender dos o más prevalece sobre la especialización y oferta en un solo servicio. La diversificación de actividades (prestación de servicios y producción, y la oferta de varios servicios) es una lógica empresarial funcional a los agronegocios y como tal, sigue intensificándose.

Esta pluriactividad y diversificación necesitan de una inversión notable en maquinaria apropiada para poder sostenerse (ver tabla 3). A pesar de ser empresas familiares, la encuesta realizada reveló que el valor de la maquinaria de la que disponían se estimaba, en promedio, en USD 1.345.552. Si a esto se le agrega el valor de las tierras, el nivel de capitalización promedio estimado de estas empresas (en máquinas y tierras) ascendía a USD 5.030.372. 
TABLA 3

Nivel de capitalización en maquinarias en función de inicio de actividad

\begin{tabular}{|c|c|c|c|c|c|}
\hline & \multicolumn{3}{|c|}{ Nivel de capitalización en maquinarias \% } & \multirow[b]{2}{*}{ Total } \\
\hline & & $\begin{array}{c}\text { Menos de USD } \\
300.000\end{array}$ & $\begin{array}{c}\text { Entre USD } \\
300.000 \text { y USD } \\
899.000\end{array}$ & $\begin{array}{l}\text { USD } 900.000 \text { o } \\
\text { más }\end{array}$ & \\
\hline \multirow{4}{*}{$\begin{array}{l}\text { Año en que } \\
\text { comenzó a } \\
\text { vender } \\
\text { servicios } \\
\text { Total }\end{array}$} & $1950-1991$ & 22,9 & 37,1 & 40,0 & $100,0(116)$ \\
\hline & $1992-2001$ & 21,6 & 41,2 & 37,3 & $100,0(169)$ \\
\hline & $2002-2016$ & 50,0 & 23,8 & 26,2 & $100,0(139)$ \\
\hline & & 31,3 & 34,4 & 34,4 & $100,0(424)$ \\
\hline
\end{tabular}

Fuente: Elaboración propia. ${ }^{*}$ Chi cuadrado de Pearson 0,034

De modo similar a lo que se expuso sobre la pluriactividad, el nivel de capitalización en maquinaria varía de modo directo en relación con la cantidad de años que se ofrecen servicios. $\mathrm{El} 40 \%$ de quienes comenzaron a vender servicios entre 1950 y 1991 estiman el valor de su maquinaria en más de USD 900.000; el 37,3 \% de quienes comenzaron a vender servicios entre 1992 y 2001 consideran alcanzar este monto con su parque de maquinarias, y solo el 34,4\% de los más nuevos estiman haber superado la barrera de los USD 900.000 en maquinaria (ver tabla 4). En el mundo de los agronegocios poder capitalizarse es un objetivo, un riesgo y un desafío, todo al mismo tiempo. Por ello, quienes poseen un mayor nivel de capitalización en maquinaria también poseen mayor capitalización en tierras, y viceversa.

TABLA 4

Nivel de capitalización en maquinarias en función del valor de las tierras

\begin{tabular}{|ll|r|r|r|}
\hline \multirow{2}{*}{} & & \multicolumn{2}{|c|}{$\begin{array}{c}\text { Nivel de capitalización en } \\
\text { maquinarias } \%\end{array}$} & \multirow{2}{*}{ Total \% } \\
\cline { 3 - 4 } & \multicolumn{2}{|c|}{$\begin{array}{c}\text { Hasta USD } \\
1.300 .000\end{array}$} & $\begin{array}{c}\text { Más de USD } \\
1.300 .000\end{array}$ & \\
\hline Valor de todas & Hasta USD 1.250.000 & 84,6 & 15,4 & $100,0(289)$ \\
sus tierras & Más de USD & 70,4 & 29,6 & $100,0(135)$ \\
Total & 1.250 .000 & 79,7 & 20,3 & $100,0(424)$ \\
\hline
\end{tabular}

Fuente: Elaboración propia. ${ }^{*}$ Chi cuadrado de Pearson: 0,135

El cuadro anterior visibiliza lo anteriormente mencionado, quienes son dueños de una mayor cantidad de tierras (o tierras por más valor) son los que poseen un mayor nivel de capitalización en maquinarias. El 15,4\% de quienes poseen tierras por hasta USD 1.250.000 poseen maquinarias por más de USD 1.300.000; este porcentaje casi se duplica (llega a 29,6 \%) entre quienes son dueños de tierras que valen más de USD 1.250.000. Estos sujetos agrarios, lejos de "vivir cómodos de sus rentas", a mayor nivel de capitalización deben trabajar más. Ello se explica, en parte, por las presiones financieras para pagar y cubrir gastos de los equipos de trabajo, en especial los contratistas de cosecha, que son quienes poseen la maquinaria más costosa (que debe ser permanentemente actualizada para poder ser competitiva). 


\subsection{La dimensión familiar como lógica articuladora de las empresas contratistas}

Se señalaba anteriormente que, en las últimas décadas, la producción de grano y la venta de servicios son actividades que coexisten dentro de la misma empresa, ya sea de manera simultánea o consecutiva en el tiempo. En este punto, la encuesta realizada visibilizó que el 33,9\% de los contratistas fue empleado de otra empresa prestadora de servicios de un familiar. El 50,6\% de las empresas tienen 1 o 2 empleados familiares, 3 o 4 empleados tienen el 28,4\% de las empresas, y el 21,0 \%, 5 o más empleados familiares. Si analizamos las composiciones societarias, podemos observar que entre las empresas que poseen más de un titular, el 66,1 $\%$ tienen una relación de familiares directos (entre al menos dos de ellos); el 19,4\% es socio de algún amigo; el 6,1 \% presentan una relación de familiares políticos entre dos socios, y el 8,3 \% tienen un socio que no entra en estas categorías.

Tanto la encuesta como el trabajo de campo realizados visibilizan que la dimensión familiar es un elemento ineludible para comprender estas empresas. La familia puede presentarse tanto como un factor estimulante como uno limitante para el desarrollo empresarial, pero nunca está ausente. Por ejemplo, cuando se preguntó a los contratistas por sus deseos respecto a la continuidad de la empresa en manos de sus hijos, el 60,6\% desea que así sea, aunque solo el 35,1\% cree que sucederá (ver tabla 5). Cuando no hay hijos varones, la continuidad es impensable. De hecho, para los casos en los que solo había hijas mujeres nadie respondió la pregunta, lo que confirma una vez más el grado de naturalización respecto a la exclusividad de lo masculino dentro de la empresa agropecuaria (se da por sentado que no corresponde contestar si no hay herederos hombres).

TABLA 5

Aspiraciones y expectativas sobre la continuidad familiar de la empresa

\begin{tabular}{|c|r|}
\hline ¿Desearía que alguno de sus hijos continuara con la empresa? & $\%$ \\
\hline Sí & $60,6(258)$ \\
No & $20,6(88)$ \\
Me da lo mismo & $16,4(70)$ \\
$2,4(10)$ \\
No es pertinente, la empresa ya no existe. & $100,0(426)$ \\
Total & $\%$ \\
\hline ¿Piensa que alguno de sus hijos continuará con la empresa? & $35,1(149)$ \\
\hline Sí & $28,0(119)$ \\
No & $35,1(149)$ \\
No sé & $1,8(7)$ \\
To es pertinente, la empresa ya no existe. & 100,0 (424) \\
\hline
\end{tabular}

Fuente: Elaboración propia.

El siguiente ejemplo ilustra el monopolio de lo masculino en el agro, que ha sido previamente analizado en otros trabajos (Manildo, 2013, Muzlera, 2009; Stølen, 2004). Claudio es un contratista que está casado y tiene dos hijos varones, también tiene una hermana, un hermano y su madre aún vive. Es del centro de la provincia de Buenos Aires (lejos de la capital). A los dieciocho años, Claudio se graduó de técnico 
electromecánico y comenzó a trabajar con su padre en la empresa que había fundado su abuelo vendiendo servicios con arados a tracción a sangre (con animales). Él es la cara visible de una empresa dedicada durante tres generaciones- a la venta de servicios de siembra y cosecha de granos como actividad principal. En noviembre de 2008 esta empresa sembraba 6.000 ha (1.500 en tierras que tomaban en alquiler o porcentaje y 4.500 en las que solo vendían el servicio), administraba campos, tenían un feed $\operatorname{lot}^{9}$ ( $\operatorname{con}^{8} 8$ terneros) y un campo con hacienda (200 vacas propias). En cuanto a capital en máquinas y herramientas, poseían siete equipos de siembra, tres de cosecha y cinco fumigadoras. Cuando el padre de Claudio falleció, la madre heredó la parte del padre (el 50 \% de la empresa), pero nunca participó de la misma. Sus dos hermanos (mujer y varón) se sumaron a la empresa familiar después de la crisis de 2001, cuando la rentabilidad y crecimiento de la empresa aumentó exponencialmente. Según su propia definición, "pasó a ser bestial". La hermana de Claudio vivía en Buenos Aires. Cuando él la convocó para sumarse a la empresa se mudó con su marido. El trabajo de ella es valorado, pero en la práctica no tiene poder de decisión. Al momento de tomar decisiones correspondientes a la empresa familiar, a pesar de ser una profesional y ser reconocida como tal, su lugar no es el mismo que el de los hombres. No obstante, al ser empresas familiares, las características y momentos en el desarrollo de la familia se entremezclan con las necesidades y posibilidades de la empresa. Este vínculo particular, a la vez que le otorga cierta flexibilidad en su funcionamiento y organización, incide claramente en el desarrollo de la empresa.

- Ella es socia y puede opinar, pero como que mucho, mucho, no sabe... en general la mayor parte de las decisiones las tomo yo y tal vez un poco con la ayuda de mi hermano. Ella se queda más en lo administrativo; ahí es bárbara, sabe mucho, nos organiza todo...

- ¿Por qué llamar a tu hermana, que vivía en Buenos Aires cuando podrías haber contratado a otro contador?

- Sí... pero mi hermana es de la familia y eso es otra cosa. Ella se esfuerza por encontrarle la vuelta para pagar menos cosas

y todo eso. Un contador de afuera nos haría gastar más plata. Y siempre sería de afuera. (Claudio, comunicación personal, noviembre de 2009)

Las tablas anteriores, al igual que los relatos de los entrevistados, muestran que la relación con la empresa es de gran relevancia y excede lo material. La empresa es un trascender, un valor que es más que una fuente de ingresos, es un legado familiar que simboliza el esfuerzo y otros valores culturales. No obstante, la posición, los roles y los aportes de las mujeres aún está muy invisibilizados e infravalorados. En este contexto cobra relevancia la importancia de lo familiar en relación con el nivel de capitalización en maquinaria de la empresa contratista. Los datos de la encuesta muestran que el $12 \%$ de quienes tienen uno o dos hijos varones tienen un nivel de capitalización en maquinarias estimado mayor a USD 1.300 .000 , mientras que el $40 \%$ de quienes tienen tres o más hijos superan este nivel de acumulación en maquinarias. Los análisis cuantitativos que estudian la relación entre cantidad de hijos varones y nivel de capitalización en maquinarias han mostrado una relación significativa entre estas dos variables. De este modo, se corrobora lo señalado con anterioridad respecto a que se establece una relación estimulante entre empresa y familia, en la que las empresas se expanden para darles lugar a los hijos varones, y a su vez la existencia de hijos varones posibilita la expansión de las empresas ${ }^{10}$ (Neiman, 2017; Muzlera, 2013).

Como puede observarse en la siguiente tabla, el porcentaje de los contratistas que desearían que sus hijos continúen la empresa aumenta a medida que aumenta el nivel de capitalización. En la tabla 6 -si consideramos que la edad mostró no tener relación con el nivel de capitalización en maquinarias, y que las hijas mujeres no son consideradas para ser parte de la empresa- se corrobora la hipótesis surgida de las observaciones en los reiterados trabajos de campo: que a mayor cantidad de hijos varones, mayor es la inversión en maquinaria. 
TABLA 6

Relación entre deseo de continuidad de la empresa y nivel de capitalización

\begin{tabular}{|l|r|r|r|r|r|}
\hline \multirow{2}{*}{$\begin{array}{c}\text { ¿Desearía que alguno } \\
\text { de sus hijos continuara } \\
\text { con la empresa? }\end{array}$} & \multicolumn{4}{|c|}{ Valor en UDS de todas sus maquinarias \% } & \multirow{2}{*}{ Total } \\
\cline { 2 - 6 } & 300.000 & $\begin{array}{c}\text { USD 300.000 } \\
\text { - USD }\end{array}$ & $\begin{array}{c}\text { > USD } \\
999.999\end{array}$ & Ns / Nc & \\
\hline Sí & 21,0 & 28,0 & 31,0 & 20,0 & $100,0(264)$ \\
No & 35,3 & 29,4 & 5,9 & 29,4 & $100,0(79)$ \\
Me da lo mismo & 22,2 & 25,9 & 40,7 & 11,1 & $100,0(79)$ \\
Total & 24,2 & 27,3 & 26,7 & 21,8 & $100,0(422)$ \\
\hline
\end{tabular}

Fuente: Elaboración propia. ${ }^{*}$ Chi cuadrado de Pearson: 0,016

Los datos de la encuesta realizada confirman lo que se señalaba con anterioridad: los hijos varones en edad de trabajar son un factor que estimula la expansión de la empresa para darles un lugar en la misma. Al igual que los chacareros (López Castro y otros, 2011; Cloquell, 2007; Barlet, 1993), las empresas que prestan servicios de maquinaria agrícola van expandiendo sus actividades para incorporar a los hijos.

TABLA 7

Relación entre cantidad de hijos y cantidad de servicios ofrecidos

\begin{tabular}{|l|r|r|r|}
\hline \multirow{2}{*}{ Cantidad de hijos varones Agrupados } & \multicolumn{2}{|c|}{$\begin{array}{c}\text { Cantidad de servicios que } \\
\text { presta (dicotómica) \% }\end{array}$} & \multirow{2}{*}{ Total } \\
\cline { 2 - 3 } & \multicolumn{1}{|c|}{ 2 o más } & \\
\hline 0 hijos & 28,9 & 71,1 & $100,0(115)$ \\
\hline o 2 hijos & 31,3 & 68,8 & $100,0(292)$ \\
3 o más hijos & & 100,0 & $100,0(18)$ \\
Total & 29,4 & 70,6 & $100,0(425)$ \\
\hline
\end{tabular}

Fuente: Elaboración propia. ${ }^{*}$ Chi cuadrado de Pearson: 0,016

Sin embargo, la familia no siempre es un elemento estimulante para la empresa prestadora de servicios. Para los empleados, dejar a la familia suele ser un factor de peso para no tomar el trabajo, y para los propietarios, ausentarse del hogar es también una causa que condiciona el tipo de servicios que está dispuesto a ofrecer (ver tabla 7). Distanciarse de la familia durante un tiempo es difícil y esta dificultad actúa como un elemento limitante a la expansión de la empresa, sobre todo, por el lado de la contratación de la mano de obra (Muzlera, 2014). Como expresa una de las entrevistadas: "Y... esta actividad no es para cualquiera. Y no me refiero solo al contratista sino también a la familia. ¿iVos sabés los meses, durante años, cuando los chicos eran chicos, que me pasaba sola en casa haciendo de madre y padre al mismo tiempo!? Y yo me la banqué" (Mónica, comunicación personal, julio 2012).

Este relato sobre las dificultades por las que atraviesa la familia no es una excepción. Hubo otros casos registrados de contratistas que culpaban a la actividad de la disolución del vínculo de pareja. Un contratista relataba: "Nunca le faltó nada, toda mi vida laburé para que estuviéramos mejor. Siempre la quise y la traté bien... y hace 2 años, a la vejez, me vino a dejar... Seis meses en casa y seis afuera no es una familia", me dijo." (Luis, comunicación personal, octubre 2009). Es una característica de los contratistas pasar muchas noches fuera del hogar. En promedio, los encuestados pasaron 69 noches fuera del hogar durante el último año por vender servicios. Sin embargo, la cantidad de noches que los contratistas han pasado fuera del hogar no es homogénea, como tampoco lo es la que pasan los titulares de las empresas y sus empleados (ver tabla 8). 
TABLA 8

Noches fueran del hogar en función del año de inicio de la actividad

\begin{tabular}{|c|r|r|r|}
\hline \multirow{2}{*}{$\begin{array}{c}\text { Año en que comenzó a } \\
\text { vender servicios }\end{array}$} & \multicolumn{2}{|c|}{ Total de noches fuera del hogar \% } & \multirow{2}{*}{ Total } \\
\cline { 2 - 3 } & Hasta 30 & 31 o más & $100,0(49)$ \\
$1950-1991$ & 80,0 & 20,0 & $100,0(178)$ \\
$1992-2001$ & 44,4 & 55,6 & $100,0(59)$ \\
$2002-2016$ & 16,7 & 83,3 & $100,0(286)$ \\
Total & 44,8 & 55,2 & \\
\hline
\end{tabular}

Fuente: Elaboración propia. ${ }^{*}$ Chi cuadrado de Pearson: 0,0109

El cuadro anterior es taxativo. Ningún contratista encuestado manifestó que le gustase dormir fuera de su casa, pero los más nuevos en el rubro son los que más necesitan hacerlo.

Aprovecho cuando tengo que hacer un trámite en el banco o algo y cada tanto me hago una escapada... Yo cada 15 o 20 días me vengo a ver los pibes y a mi señora. Imaginate, tengo uno de 7 y uno de 4 ... la otra vez que me vine, el de 4 me vio y pegaba unos saltos de alegría hasta acá. Si cuando me voy se llega a romper una máquina, me llaman por teléfono y con las chatas de ahora a 140 o $160[\mathrm{Km} / \mathrm{h}]$ en unas 6, 7 u 8 horas llegás a cualquier lado. (Pedro, comunicación personal, julio de 2009).

Los empleados se ausentan de sus hogares durante toda la campaña en el caso de que trabajen para una empresa que geográficamente se desplace a distintos lugares para realizar las labores culturales. ${ }^{11}$ En cambio, si la distancia es menor, el alejamiento del hogar se reduce a pocos días o a ninguno. Baltasar, con 31 años, casado y con dos hijos ( 5 y 3 años), hablando al respecto expresaba lo siguiente:

En la cosecha te pagan mucho más. Capaz que con lo que sacás en una temporada de cosecha te hacés lo mismo que durante todo el año con los Gutiérrezy no tenés que estar tirando de la maleta ni cambiando caños entre el barro, pero yo laburando para paperos vengo a casa todas las noches, veo a los nenes y a mi señora, y los domingos, en general, tengo vida. Del otro modo ¿Sabés lo que es estar meses sin ver a tu familia?! Mientras el cuerpo me aguante me quedo con esto (Baltasar, comunicación personal, noviembre de 2011).

Otro factor determinante es el servicio prestado. Por vender servicios de cosecha, en promedio, los encuestados durmieron fuera del hogar 54,43 días. Por prestar servicios de pulverización, 25,35 días, y por prestar servicios de siembra, 24,52 días. Estos datos indican que, a pesar de la tendencia homogeneizante de la oferta, hay especificidades que aún no han desaparecido. Como se señalaba anteriormente, los contratistas de cosecha fueron los primeros en "popularizarse" en el medio y eran empresarios, en general del norte de la Región Pampeana, que comenzaban la cosecha de granos al norte y avanzaban hacia al sur; estaban varios meses fuera del hogar, pero no eran productores. La oferta de siembra y pulverización, como actividad terciarizada, se hizo común con el paquete soja RR, siembra directa, glifosato después de 1996, y fueron productores locales que diversificaron su actividad y comenzaron a vender servicios a vecinos (Muzlera, 2013). Otro dato a considerar es que el costo y la complejidad logística de movilizar un equipo de cosecha es superior al de movilizar uno de pulverización o uno de uno de siembra. Un dato interesante que nos revela la encuesta realizada indica que entre aquellos que poseen maquinarias por mayor valor (más de USD 1.300.000), el 60,0 \% duermen fuera del hogar en promedio 30 días al año (ver tabla 9). Entre quienes poseen menos de USD 1.300 .000 en maquinarias "solo" el 44,9\% debe dormir fuera de su casa treinta noches por prestar servicios de cosecha. 
TABLA 9

Noches que durmió fuera del hogar por vender servicios de cosecha

\begin{tabular}{|l|r|r|r|}
\hline \multirow{2}{*}{$\begin{array}{c}\text { Nivel de capitalización en } \\
\text { maquinarias }\end{array}$} & \multicolumn{2}{|c|}{$\begin{array}{c}\text { Cantidad de días que (por vender } \\
\text { servicios de cosecha) durmió fuera } \\
\text { del hogar. \% }\end{array}$} & \multicolumn{2}{|c|}{ Total } \\
\cline { 2 - 3 } & Hasta 30 días & Más de 30 días & $100,0(218)$ \\
\hline Hasta USD 1.300.000 & 55,1 & 44,9 & $100,0(94)$ \\
Más de USD 1.300.000 & 40,0 & 60,0 & $100,0(312)$ \\
Total & 52,4 & 47,6 & \\
\hline
\end{tabular}

Fuente: elaboración propia. ${ }^{*}$ Chi cuadrado 0.049

Otra dinámica que explica en gran medida el peso de lo familiar en la reproducción familiar-empresarial es el proceso de convertirse en socio. Cuando el o los dueños de una empresa de venta de servicios tienen hijos varones (y se logra consenso sobre la continuidad familiar de la empresa), él o los hijos, de quienes se prevé que en el futuro continuarán la empresa, comienzan a trabajar en la misma desde muy jóvenes, en general entre los 15 y los 18 años, y muchas veces abandonan su educación formal. Comienzan “ayudando", después se convierten en empleados y finalmente se transforman en socios. Es muy raro que los socios sean más de tres. El 88,9 \% de las empresas están compuestas de hasta tres socios (33,3\% tienen un solo dueño; 42,1\% dos socios, y el 13,5 \% tres socios). Cuando los hijos se transforman en socios de los padres la sociedad actual se divide y cada antiguo socio toma un nuevo rumbo (ver tabla 10).

TABLA 10.

Relación empleados familiares / empleados totales

\begin{tabular}{|l|r|r|}
\hline & \% válido & \multicolumn{1}{|c|}{ \% acumulado } \\
\hline Hasta 0,25 (incluido) & $25(72)$ & 25,0 \\
Más de 0,25 hasta 0,39 (Incluido) & $25(72)$ & 50,0 \\
Más de 0,39 hasta 0,50 (Incluido) & $30,5(87)$ & 80,5 \\
Más de 0,50 & $19,5(56)$ & 100,0 \\
Total & $100,0(287)$ & \\
\hline
\end{tabular}

Fuente: Elaboración propia.

Como se refleja en la tabla anterior, el $25 \%$ de las empresas tiene como familiares de los dueños al 25 $\%$ de los empleados, y otro $25 \%$ de los contratistas tienen entre el $25 \%$ y el $39 \%$ de sus empleados a familiares. El 30,5\% de las empresas tienen entre un $39 \%$ y un $50 \%$ de empleados familiares, y el 19,5 $\%$ de las empresas contratistas tienen más del $50 \%$ de sus empleados familiares. En el contexto familiarempresarial presentado, toda esta superposición de los espacios productivos con los reproductivos, con todo lo que ello implica (yuxtaposición de las economías del hogar y la empresa, dinámicas organizacionales, inversiones en maquinaria, autos familiares, camionetas que cumplen ambas funciones, vacaciones versus inversión, estudio de los hijos versus expansión de la empresa, etc.), sigue siendo una característica habitual en el campo argentino, que, a pesar de la continua y creciente capitalización de sus contratistas, continua teniendo un factor articulador relevante como lo es la familia.

\section{CONSIDERACIONES FINALES}

En congruencia con el paradigma agronegocio y la producción en red, el subsector de los contratistas es altamente dinámico, flexible y adaptable. Esto para nada implica que estas características redunden en el 
bienestar de estos sujetos. Como decía Sennett (2000), la fortaleza de la red reside en la debilidad de sus nodos. Esta lógica productiva es eficiente a costa de los perjuicios y dificultades que causa en los sujetos que la forman parte. En este sentido, el dinamismo al que se hace referencia en este trabajo contempla la pluriactividad y la gran capacidad de adaptación a las condiciones técnicas, político-económicas y de mercado, que se modifican vertiginosamente, y fuerzan los proyectos de biografías de estos sujetos. Los contratistas o prestadores de servicios de maquinaria agrícola son sujetos sobre los que se observa cómo el paradigma del agronegocio ejerce su poder de transformación. El agronegocio transforma empresas, los servicios que ofrecen, y sus dinámicas de organización. El ejemplo más claro de ello es que más de las tres cuartas partes de estos son pluriactivos (combinan la venta de servicios con la producción agropecuaria o venden más de un servicio).

Los resultados de la encuesta y el trabajo de campo realizados, en concordancia con otros trabajos anteriores, muestran cómo las identidades de las generaciones de más edad (chacareros, cosecheros, ganaderos) se van perdiendo y dejando lugar a la de empresarios. El rasgo observado más relevante es que la actividad ya no los define, o lo hace cada vez menos. Entre los más jóvenes, sembrar soja, vender servicios de siembra o pulverización es casi anecdótico. La actividad ofrecida, como se ha podido observar a lo largo del texto, suele yuxtaponerse con otras y se define según las oportunidades de negocios.

En conjunto, las empresas encuestadas poseen altos niveles de capitalización y flexibilización, y es la dimensión familiar la característica articuladora más persistente de un modelo anterior. A pesar de que esta dimensión también muestra signos de transformación (sobre todo porque se administra la economía de la empresa separada de la del hogar), las estrategias de la empresa están supeditadas al ciclo de vida familiar y a la composición de la familia (sexo de los hijos, edad, número de hijos, etc.). Esta expansión suele tener el límite de las lógicas familiares. Lo común es que los socios sean hermanos o amigos, a veces otros parientes, pero de una misma generación. Cuando se incorporan las generaciones más jóvenes a la sociedad empresarial, esta se modifica en su composición. Las sociedades, excepto situaciones puntuales, se dan entre padre e hijos, hermanos, parientes y amigos. Es muy difícil encontrar una sociedad que haya estado constituida por dos hermanos o dos amigos y que estos permanezcan siendo socios si se incorporan los hijos de alguno o de ambos. También es casi imposible encontrar una sociedad sostenida solo por el objetivo económico. Lo doméstico sigue siendo de peso para entender a estos empresarios.

Otro rasgo que se ha modificado muy poco es el lugar de la mujer en las empresas. En las dinámicas familiares, el empoderamiento de las mujeres va lentamente en aumento, pero las empresas -desde lo formalsiguen siendo territorio masculino. En particular, las empresas, a pesar de aumentar los niveles de racionalidad formal en su administración para lograr mayores niveles de eficiencia, no tienden a especializarse, sino todo lo contrario. Los prestadores de servicios tienden a diversificar el tipo de oferta. Empresas que antes tenían tres cosechadoras han vendido una o dos para incorporar siembra y/o pulverización. Otras empresas, que se dedicaban a la agricultura, han incorporado servicios de siembra y grandes contratistas han incursionado en la agricultura. Estas decisiones, como se vio en las entrevistas, se fundamentan en los vínculos personales que se establecen con los empleados, las necesidades de los hijos varones de ingresar al mercado de trabajo, o con estrategias de desinversión para cubrir algún gasto familiar.

La pluriactividad y diversificación tensionan, así, con el deseo de continuidad empresarial, en tanto legado familiar. El cálculo racional para aumentar la eficacia empresarial choca con valores y prácticas que responden a otras lógicas y que van perdiendo peso. No obstante, en los relatos de los entrevistados, el esfuerzo sigue siendo un valor y el sostén de la empresa -cuando esta actividad precedió a la de productor-. Estamos así ante la presencia de sujetos sumamente funcionales a un agro globalizado y en transición: flexibles, dispuestos a esforzarse y arriesgarse, individualistas, competitivos y empeñados en persistir.

En resumen, podemos observar cómo el contratismo no solo fue -y es- funcional a los productores demandantes de sus servicios, porque les brindaba una alternativa productiva y modernizante que de otra manera los excluiría, sino que lo fue -y es- también como una actividad a veces complementaria y otras directamente alternativa a una empresa agropecuaria que se había tornado inviable. En este contexto, seguir 
siendo contratista es una apuesta arriesgada que puede generar tanto angustia como ansiedad y adrenalina, poniendo en tensión los valores familiares heredados con los modelos empresariales "modernos y eficientes".

\section{ReFERENCIAS}

Agüero, R. O., Rivarola, A., y Maldonado, R. A. (2007). Caracterización del contratismo de servicios en un sector de la pampa cordobesa: las localidades de Alcira Gigena y Berrotarán. Presentación de resultados preliminares de investigación. Mundo Agrario, 7(14), 1-28. Recuperado de: https://dialnet.unirioja.es/servlet/articulo?codigo= 4500689

Azcuy Ameghino, E. (2009). El papel del contratismo de servicios de maquinaria en la caracterización socioeconómica de las pequeñas explotaciones agropecuarias. Realidad Económica, (244), 26-36. Recuperado de: http://www.ia de.org.ar/articulos/el-papel-del-contratismo-de-servicios-de-maquinaria-en-la-caracterizacion-socioeconomica

Barsky, O., y Gelman, J. (2009). Historia del agro argentino. Desde la conquista hasta comienzos del siglo XXI. Buenos Aires: Sudamericana.

Barsky, O., y Pucciarelli, A. (1997). El agro pampeano. El fin de un periodo. Buenos Aires: FLACSO.

Barlett, P. (1993). American Dreams, rural realities: family farms in crisis. Studies in rural culture. Chapel Hill: The University of North Carolina Press.

Baumeister, E. (1980). Estructura agraria ocupacional y cambio tecnológico en la región maicera. La figura del contratista de máquinas. Documento de Trabajo $\mathrm{N}^{\circ} 10$. Buenos Aires: CEIL.

Bonanno, A., y Wolf, S. (2018). Resistance to Neoliberal Agri-Food: A Critical Analysis. New York: Routledge.

Bonanno, A. (2017). The Legitimation Crisis of Neoliberalism. The State, Will-Formation and Resistance. New York: Palgrave-Macmillan.

Bonanno, A., Martínez, F., y Aboites, G. (2016). El Neoliberalismo un Momento de la Globalización. Mexico D.F: Fontamara.

Bourdieu, P. (1998). La distinción. Criterios y bases sociales del gusto. Madrid: Taurus.

Cloquell, S. (Coord.) (2007). Familias rurales. El fin de una historia en el inicio de una nueva agricultura. Buenos Aires: Homo Sapiens.

Craviotti, C. (2002). Pampas family farms and technological change: strategies and perspectives towards genetically modified crops and no-tillage systems. IJSAF 10(1), 23-30. Recuperado de: https://docs.wixstatic.com/ugd/ efa020_74f7a697a73c4bd897e533b7066f 7780.pdf

Craviotti, C., y Gras, C. (2006). De desafiliaciones y desligamientos: trayectorias de productores familiares expulsados de la agricultura pampeana. Desarrollo Económico, 46(181), 117-134. Recuperado de: https://www.jstor.org/ stable/4151103?seq=1\#page_scan_tab_contents

Devoto, R. (1989). Contratistas de servicio y contratistas de producción en la visión de los años 80. Carpeta de economía agrícola. Carpeta de economía agrícola. Temas de investigación, 39. Pergamino: INTA Estación experimental regional agropecuaria.

Devoto, R. (1988). Consideraciones acerca del desempeño de productores-contratistas en los partidos de Rojas y Pergamino. Carpeta de economía agrícola. Temas de investigación, 35. Pergamino: INTA Estación experimental regional agropecuaria.

Fernández, D. (2013). Vinculaciones entre los procesos de cambio tecnológico y concentración económica en la agricultura pampeana en la década del '90'. Documentos del CIEA, (8), 99-114. Recuperado de: http://www.cie a.com.ar/web/wp-content/uploads/2016/11/Doc8_6.pdf

Fernández, D. (2017). El trabajo en la chacra. Sobre los efectos de la difusión del contratismo de servicios agrícolas en las posibilidades de subsistir de la producción familiar pampeana. En J. Muzlera y M. Poggi (Comps.), Poder, Conflicto y Trabajo en el Agro Argentino (pp. 155-180). Buenos Aires: CICCUS.

Forni, F., y Tort, M. I. (1991). De chacareros a 'Farmers contratistas'. Serie documentos de trabajo 25. Buenos Aires: CEIL. 
Garbers, R. (2016). Presente y Futuro de la Mecanización Agricola en América Latina. Conferencia realizada en el Congreso IICA 2016 (pp. 1-4). Washington DC. Recuperado de: http://www.iica.int/sites/default/files/ events/presentations/2016-11/agenda_-_iica-ifpri_seminario_espanol_0.pdf

Gras, C., y Hernández, V. (Coords.) (2009). La Argentina rural. De la agricultura familiar a los agronegocios. Buenos Aires: Biblos.

Gras, C., y Hernández, V. (Coords.) (2013). El agro como negocio: producción, sociedad y territorios en la globalización. Buenos Aires: Biblos.

Gras, C., y Hernández, V. (Coords.) (2016). Radiografía del nuevo campo argentina. Del terrateniente al empresario transnacional. Buenos Aires: Siglo XXI editores.

Guber, R. (2001). La etnografía. Método, campo y reflexividad. Bogotá: Norma.

Hammersley, M., y Atkinson P. (1994). Etnografía. Métodos de investigación. Barcelona: Paidós.

Hernández, V., y Muzlera, J. (2016). El contratismo y su integración al modelo de agronegocios: producción y servicios en la región pampeana. Mundo Agrario 17(34), 1-22. Recuperado de: https://dialnet.unirioja.es/ servlet/ articulo?codigo $=5598019$

Kahneman, D. (2003). A psychological perspective on economics. The American Economic Review, 93(2), 162-168. Recuperado de: http://ase.tufts.edu/gdae/cs/Psychological\%20Perspective.pdf

Kay, C. (2016). La transformación neoliberal del mundo rural: procesos de concentración de la tierra y del capital y la intensificación de la precariedad del trabajo. RELAER, 1(1), 1-26. Recuperado de: https://core.ac.uk/down load/pdf/72002752.pdf

Llovet, I. (1991). Contratismo y agricultura. En O. Barsky (Ed.), El desarrollo agropecuario pampeano (pp. 607-665). Buenos Aires: INDEC, INTA e IICA.

Lódola, A. (2008). Contratistas, cambios tecnológicos y organizacionales en el agro argentino. Buenos Aires: CEPAL.

Lódola, A., Angeletti, K., y Fossati, R. (2005). Maquinaria agrícola, estructura agraria y demandantes. Colección Cuadernos de Economía 72. Buenos Aires: Ministerio de Economía de la Provincia de Buenos Aires.

Lombardo, P., y Marra, R. (2018). Economías de escala y externalización de labores agrícolas en la región pampeana (Argentina).Perspectivas Rurales Nueva Época, 16(31), 143-153. https://doi.org/10.15359/prne.16-31.8

López Castro, N. y Prividera, G. (2011). Repensar la agricultura familiar: aportes para desentrañar la complejidad agraria pampeana. Buenos Aires: CICCUS.

Manildo, L. (2013). La identidad chacarera en las grietas del paisaje sojero. Desplazamientos, transmisiones y apropiaciones intergeneracionales en las transformaciones recientes de la producción familiar pampeana. Buenos Aires: Imago Mundi.

Murmis, M. (1998). El agro argentino: algunos problemas para su análisis. En N. Giarraca y S. Cloquell (Comps.), Las agriculturas del Mercosur: el papel de los actores sociales (pp. 205-248). Buenos Aires: La Colmena/CLACSO.

Muzlera, J. (2009). Chacareros del siglo XXI. Herencia, familia y trabajo en la Pampa Gringa. Buenos Aires: Imago Mundi.

Muzlera, J. (2013). La modernidad tardia en el agro pampeano: sujetos agrarios y estructura productiva. Bernal: UNQ.

Muzlera, J. (2014). Estrategias y motivaciones de capitalización entre contratistas de maquinaria agrícola pampeana. Papeles de Trabajo 8(13), 250-270. https://ri.conicet.gov.ar/handle/11336/42660

Muzlera, J. (2016). El peso de las familias en las empresas de contratistas de maquinaria agricola pampeanos. Ponencia presentada en las IX Jornadas de sociología de la UNLP 2016. No publicado. La Plata: UNPL

Neiman, M. (2017). La herencia en las empresas familiares de la región pampeana argentina durante el actual período de auge económico de la actividad agrícola. Papers, 102(3), 509-531. http://dx.doi.org/10.5565/rev/papers.2269

Paz, R. (2017). Las grietas de los agronegocios y los imperativos de la agricultura familiar: hacia una perspectiva conceptual. Revista Latinoamericana de Estudios Rurales, 2(3), 39-63. Recuperado de: http://www.ceil-conicet .gov.ar/ojs/index.php/revistaalasru/article/view/194/117

Peretti, P. (2013). Chacareros, soja y gobernabilidad. Del Grito de Alcorta a la Resolución 125. Buenos Aires: CICCUS. 
Piñeiro, V., Robles, M., y Elverdin, P. (2017). El Agro Argentino. Un Sistema Productivo y Organizacional Eficiente. IFPRI Discussion Paper 1621SP. Washington, D.C.: International Food Policy Research Institute (IFPRI). Recuperado de: http://ebrary.ifpri.org/cdm/ref/collection/p15738coll2/id/131103

Pizarro, J., y Cacciamani, M. (1980). Características descriptivas de los "contratistas" en maquinaria agrícola. Carpeta de Economía Agrícola. Temas de investigación, 10. Pergamino: INTA Estación experimental regional agropecuaria.

Sennett, R. (2000). La corrosión del carácter. Las consecuencias personales del trabajo en el nuevo capitalismo. Barcelona: Anagrama.

Stadler, S., y Botta, G. (2015). Caracterización parcial de los contratistas de servicios de maquinaria agrícola en la micro región 2, provincia de La Pampa, Argentina. Revista de la Facultad de Ciencias Agrarias, 47(2), 135-143. Recuperado de: http://revista.fca.uncu.edu.ar/index.php?option=com_contentyview=articleyid=333:2015-1 0-23-18-26-53ycatid $=21: 2015-10-22-17-24-20$ yItemid $=27$

Stølen, K. A. (2004). La decencia de la desigualdad. Género y poder en el campo argentino. Buenos Aires: Antropofagia.

Tort, M. I. (1983). Los contratistas de maquinaria agrícola: una modalidad de organización económica del trabajo agrícola en la pampa húmeda. Documento de trabajo 11. Buenos Aires: CEIL.

Ullua, A. (2017). Contrato de maquinaria agricola en el noroeste de la provincia de Buenos Aires. Ponencia en el IV Congreso Nacional de Derecho Agrario Provincial. Salta, Argentina: UNPL/SEDICI. Recuperado de: http:// sedici.unlp.edu.ar/handle/10915/60450

Urcola, H. A., De Sartre, X., Veiga, I., Elverdin, J., y Albaladejo, C. (2015). Land Tenancy, Soybean, Actors and Transformations in the Pampas: A District Balance. Journal of Rural Studies, (39), 32-40. https://doi.org/10. 1016/j.jrurstud.2015.03.001

Valles, M. (2002). Entrevistas cualitativas. Cuadernos metodológicos 32. Madrid: CSIC.

Van Dijk, T. (2003). Ideología y discurso. Barcelona: Ariel.

Varela, J. C., y Cappello, J. E. (2018). Caracterización de los contratistas de servicios de maquinaria agrícola-localidad de Quemú Quemú, La Pampa. Semiárida, 26(1), 43-52. Recuperado de: http://www.biblioteca.unlpam.edu.ar /pubpdf/rev-agro/v26n1a20varela.pdf

Villulla, J. M. (2015). Las cosechas son ajenas. Historia de los trabajadores rurales detrás del agronegocio. Buenos Aires: Cien Flores.

\section{Notas}

1 Problemáticas que estos sujetos sociales comparten en un mundo globalizado. De esta forma, la globalización remite a la reorganización social del tiempo y del espacio, es decir, a la manera como estas dimensiones afectan la vida en las sociedades contemporáneas. Una de las características principales de esta globalización es la financiarización, es decir, el predominio del sector financiero sobre el sector productivo. La financiarización, proceso muy extendido que provoca, entre otras cosas, un conflicto entre capital financiero y productivo, porque las lógicas del capital financiero son cortoplacistas y condicionan la producción de las empresas que necesariamente deben orientar sus estrategias con una mirada de más largo plazo, necesitan transformarse o acaban saliendo del juego (Bonanno y Wolf, 2018; Bonanno, 2017; Bonnano, Martínez y Aboites, 2016).

2 Los de cosecha datan de fines de siglo XIX (Barsky y Gelman, 2009, p. 210) y desde ese entonces esta labor ha sido la más terciarizada. En 1988 el 49 \% de las explotaciones pampeanas contrataban algún servicio, en 2002 lo hacían el 75 $\%$ de las explotaciones. Entre 2001-2002 y 2004-2005, para la provincia de Buenos Aires, la superficie trabajada por contratistas aumentó $9 \%$ para siembra convencional, $112 \%$ para siembra directa y $38 \%$ para cosecha. En 2012 entre el $70 \%$ y el $85 \%$ de las superficies implantadas con los 5 principales cultivos, 31.000 .000 ha, fueron trabajadas a cargo de terceros (Peretti, 2013, p. 37). Desde la Dirección de Contratismo e Insumos Agrícolas se estimaba que los prestadores de servicios agropecuarios sembraban el $60 \%$ de los granos de la argentina, pulverizan el $75 \%$ y que levantan el $90 \%$ (Garbers, 2016).

3 La rápida y profunda concentración de la tenencia de las tierras productivas, favorecida por la liberación de la soja RR al mercado (en 1996) y la devaluación de la moneda (en 2002), provocó un gran aumento en los valores de los alquileres. A modo ilustrativo podemos señalar que una hectárea en el sudeste bonaerense, que en 1995/1996 se alquilaba a 140 USD/ Año, en 2008/2009 estaba 500 USD/Año. El acaparamiento y pérdida de tierras producto de este gran dinamismo del 
mercado presentaba a la oferta de servicios (por parte de los exproductores) y a la contratación de labores (por parte de los concentradores de la tenencia de la tierra y la producción) como la mejor elección.

4 Las labores culturales más comunes son: cosecha, pulverización y siembra directa (Garbers, 2016).

5 A nivel nacional solo existía el Relevamiento Provincial de Servicios Agropecuarios 2002 (RPSA) llevado a cabo por la Dirección Provincial de Estadística de la Provincia de Buenos.

6 Son empresas orientadas al mercado, para las que el lucro es el principal estímulo de su funcionamiento (aunque no el único), con capacidad de acumulación, y que se desarrollan vis a vis con las necesidades y etapas del ciclo de vida de la familia a la que pertenecen.

7 Los datos demográficos de la encuesta realizada muestran que el promedio de edad de los contratistas es de 47,13 años. El $25 \%$ tiene hasta 39 años, el $50 \%$ hasta 46 y el $75 \%$ hasta 56 años. Cuando se les preguntó por el tamaño de la localidad en la que viven, el 15,0 \% contestó que vive en el campo; el 23,3 \% en pueblos de hasta 5.000 habitantes; el 18,9 en pueblos de entre 5.000 y 10.000 habitantes; el $25 \%$ en ciudades de hasta 40.000 habitantes; el 13,3\% en ciudades de más de 40.000 habitantes y menos de 500.000 y el 4,4\% restante vive en ciudades de más de medio millón de habitantes. A la pregunta en qué provincia viven, el 37,1 \% respondió Provincia Buenos Aires; el 28,1\% en Santa Fe; el 15,6\% en Córdoba; el 7,8\% en Entre Ríos; el 2,4\% en la Pampa y el resto de las provincias albergan entre el 0,6\% y el 2,0\% de los contratistas cada una.

8 Se refiere a máquinas para diversas labores culturales (siembra, cosecha y fumigación).

9 Es un anglicismo, de uso común Argentina, para designar la cría de vacunos en corrales de engorde, no a campo. El ganado recibe el alimento balanceado en comederos. La técnica de feedlot surgió ante la necesidad de intensificar la producción por el alto valor que los campos adquirieron con el aumento del precio de la soja y la posibilidad de expandir la frontera agrícola gracias a las nuevas técnicas agrícolas y variedades transgénicas.

10 No obstante, esta relación no fue significativa cuando se analizó la relación entre cantidad de hijos y capitalización total (máquinas y tierras).

11 Es casi imposible establecer a partir de cuántos kilómetros se vuelve o no al hogar. Sí es claro, en cambio, que los dueños que están a una distancia relativamente pequeña (menos de 90 minutos en camioneta, aproximadamente) vuelven al hogar cada noche, algo que no suelen permitir a los empleados. Que los empleados duerman en el campo "los estimula" a trabajar desde más temprano y hasta más tarde, y permite cuidar las máquinas por la noche. No obstante, esto varía según los empresarios, los arreglos con los empleados y la "peligrosidad de robo de maquinarias" de la zona. 